\section{A) Check for updates}

Cite this: Nanoscale, 2020, 12, 19870

\title{
Simulating facet-dependent aggregation and assembly of distributions of polyhedral nanoparticles $\uparrow$
}

\author{
George Opletal, (D) *a Shery L. Chang ${ }^{b}$ and Amanda S. Barnard (iD) ${ }^{c}$ \\ Coarse-grained molecular dynamics simulations of diamond nanoparticles were performed to investigate \\ the effects of size polydispersity on three polyhedral shapes chosen to span a diverse space of surface \\ interactions. It was found that the resulting self-assembly was size dependent as the simulations were \\ quenched, with the largest nanoparticles providing a clustered scaffold for subsequent smaller nano- \\ particle assembly. Additionally, facet-facet interactions were dominated by the $\{111\}$ surface and the \\ resulting aggregate was dominated by meso-sized porosity for monodisperse systems, broadening to \\ larger diameters for polydisperse systems.
}

Received 4th May 2020,

Accepted 28th August 2020

DOI: $10.1039 / \mathrm{d} 0 \mathrm{nr} 03470 \mathrm{c}$

rsc.li/nanoscale

\section{Introduction}

Individual nanoparticles are often credited with remarkable properties exploited in applications including catalysis, drug delivery, optoelectronics and composites, but individual nanoparticles tend to aggregate into clusters up to several microns in size ${ }^{1-3}$ While this is often considered a hindrance, motivating numerous processes designed to separate aggregates into primary nanoparticles, ${ }^{4-6}$ the size, porosity, strength and complexity of aggregates play an important role in determining reactivity, toxicity, transport and efficiency ${ }^{7-13}$ and can present us with a new range of degrees of engineering freedom to create application-specific "designer" nanoparticle-based systems.

Many of the functional properties of nanoparticle systems are due to the collective behaviour or order of multiple nanoparticles, rather than the properties of the individual nanoparticles themselves. Examples include the tribological properties of powders ${ }^{14}$ the toxicological properties of colloids, ${ }^{15}$ the mechanical properties of composites, ${ }^{16}$ the collective electromagnetic properties exhibited in surface plasmon resonances and nanoporous materials formed via aggregation or self-assembly. ${ }^{17}$ The superstructure of the aggregate, characterised by the number density, the porosity and/or the degree of

\footnotetext{
${ }^{a}$ Data61 CSIRO, Door 34 Goods Shed Village St, Docklands, Victoria, Australia. E-mail: george.opletal@data61.csiro.au

${ }^{b}$ Electron Microscopy Unit, Mark Wainwright Analytical Centre, and School of Materials Science and Engineering, University of New South Wales, Sydney, NSW 2052, Australia

${ }^{c}$ ANU Research School of Computer Science, Acton, ACT 2601, Australia $\dagger$ Electronic supplementary information (ESI) available. See DOI: 10.1039/ d0nr03470c
}

local or long-ranged order, is often the design parameter responsible for performance. This is certainly the case in nanodiamond-based drug delivery platforms, where the structure of nanodiamond aggregates is used to tune the dose and dose rate chemotherapeutic delivery, ${ }^{18-23}$ as well as some other treatments. ${ }^{24-26}$ The optimisation of platforms such as these requires a detailed understanding of the global structure of an aggregate as a whole and the local structure surrounding each nanoparticle (including inter-particle interfaces and "free" facets) and the ability to predict these parameters based on the sample composition. Much like a recipe, we need to be able to anticipate the outcome when we mix and relax a set of nanoparticle "ingredients".

Predicting the meso-structure of aggregated nanoparticles is an ideal problem for computer simulations (that can control the sample composition and characterise the outputs exquisitely), but historically the aggregation of large systems of nanoparticles has required practical simplification of a variety of parameters. Nanoparticles challenge the limits of colloid science due to their small size, variable shape and complex surface structure. In the past, the majority of studies on the impact of nanoparticle aggregation had begun by applying colloid science principles, based on the classical DerjaguinLandau-Verwey-Overbeek (DLVO) theory, ${ }^{27-29}$ and focused on qualitative interpretation of the observed agglomeration behavior. ${ }^{11,30}$ However, numerous important factors, such as steric, geometric, hydrodynamic and hydration, are not considered by the classical DLVO theory and can have an importance that can impact nanoparticle agglomeration. In short, the diverse properties of the various facets, which can be exposed surfaces or enclosed interfaces, need to be included.

Alternatively, quantitative simulations can account for the surface science of the particle-particle interactions, and dyna- 
mical studies have reported details of the agglomeration kinetics and agglomeration morphology of a variety of nanoparticles. This includes the use of computationally inexpensive methods, such as classical molecular dynamics, ${ }^{31}$ Brownian dynamics $^{32}$ and Monte Carlo methods; ${ }^{33-36}$ but many of these types of studies approximate nanoparticles as either homo-

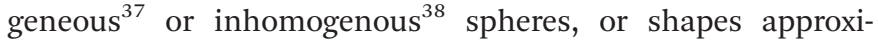
mated by strategically placed spheres. ${ }^{39}$ Chemical and geometric realism is often sacrificed to accommodate the larger number of nanoparticles required to capture long-range effects and sufficient statistical diversity to overcome the impact of the imposed translational symmetry of the simulation environment. Ideally, we need to reintroduce more of this realism, such as polyhedral shapes, heterogeneous surface properties and nanoparticle specific interactions, without giving up the advantages afforded by approximate simulation methods.

A convenient compromise can be achieved by adding more details and heterogeneity to the surface of coarse-grained nanoparticles. Recently, a new software package has been released to model the Simulated Nanoparticle Assembly with Protoparticles (SNAP), ${ }^{40,41}$ and overcome the need to make approximations regarding the nanoparticle size and shape, and to eliminate assumed monodispersity. This package is capable of modelling the aggregation of a large number of complex polyhedra of any size, accounting for heterogenous facet-dependent interactions and allowing for distributions of sizes. This package was designed to allow users to define any polyhedral shape and then automatically decorate the surface with protoparticles encoded with specific types of user-defined physicochemical properties.

In the present study, we have used the SNAP package to investigate the impact of size distributions on the meso-structure of aggregated faceted polyhedral nanodiamonds in a vacuum. We have used this approach previously ${ }^{57}$ to gain useful insights into experimental self-assembly in aqueous solutions by hypothesizing that the assembled structures resulted from a competition between the short-ranged electrostatic forces and solvent-mediated forces. Here, we focus solely on the assembly resulting from the facet dependent electrostatic interactions mediated by having pristine facets. As mentioned above, the applications of this material depend on the properties of the entire aggregate (as well as individual nanoparticles), but they also represent an ideal case study for a variety of other reasons. They are known to form tight aggregates or self-assembled filaments, depending on the storage conditions. $^{42,43}$ The polyhedral shapes are known to have low index facets with distinctly different chemical structures (aromatic or aliphatic) and electrostatic surface potentials (positive, negative or near neutral). ${ }^{44}$ The multi-polar facet-dependent surface electrostatic potential has implications and their use $^{45}$ is responsible for the aggregation and self-assembly. ${ }^{46,47}$ From a computational perspective this is interesting since these properties can be calculated, ${ }^{48}$ and the interaction between different combinations of surface facets can be used to parameterise SNAP. ${ }^{49,50}$ As we will see, the availability of surface facets on lining the inside of pores, and the overall ordering of the aggregate, is strongly affected by the size of the positive (aliphatic) and negative (aromatic) facets. The porosity and packing efficiency can be affected by including polydispersity with the smaller nanoparticles likely to line the inside of the pores regardless of the nanoparticle shape.

\section{Methods}

SNAP is divided into three programs that represent the various stages of preparing, running and analysing a given simulation. ${ }^{40,41}$ The Generator builds user-defined nanoparticle sizes and shapes, prepares the surface mesh to describe the surface properties, and establishes the initial configuration of nanoparticles in the simulation cell. The Simulator takes in the initial configuration, potential parameters and simulation conditions defined by the User to run molecular dynamics (MD). The Analyser takes the outputs of the simulation and performs some basic structural, numerical and statistical analyses.

\section{Sample generation}

A SNAP simulation requires the input of an initial configuration of nanoparticles along with their velocities. A nanoparticle is generated by defining its facets using a collection of normal vectors defining each of the nanoparticle's facet planes, akin to the Wulff construction. The surface enclosed by the intersection of these facet planes is calculated and represented by the generation of an equidistant surface mesh of interaction points known as protoparticles. The protoparticles decorating different facets are encoded with different physicochemical properties (as mentioned above) and are the origins of the force fields describing the interactions between nanoparticles. A surface mesh density of 0.2 protoparticles per $\AA^{2}$ has been used in this study to stop nanoparticle overlap and is governed by the range of the repulsive part of the Morse potential, as described elsewhere. ${ }^{41}$ The mass of each nanoparticle is equally distributed over the surface protoparticles so that the subsequent simulation represents an approximation where the moment of inertia differs from a realistic mass distribution. Once the protoparticle meshes are generated for each nanoparticle shape, the nanoparticles are duplicated into a simulation cell on a grid to produce a required number density. Random initial rotations, random initial switching of nanoparticles of the chosen sizes, and an initial high-temperature MD equilibration then randomize the starting configuration, eliminating the initial grid.

In the present study, we have used complex polyhedra enclosed by low index $\{100\},\{110\}$ and $\{111\}$ facets, known to have characteristic positive, near-neutral and negative surface electrostatic potentials, respectively. The three polyhedra included here are the great rhombicuboctahedron (GRO), which is dominated by positive $\{100\}$ surface area (Fig. 1(a)); the small rhombicuboctahedron (SRO), which is dominated by near-neutral $\{110\}$ surface area (Fig. 1(b)); and the modified truncated octahedron (mTO), which is dominated by negative 

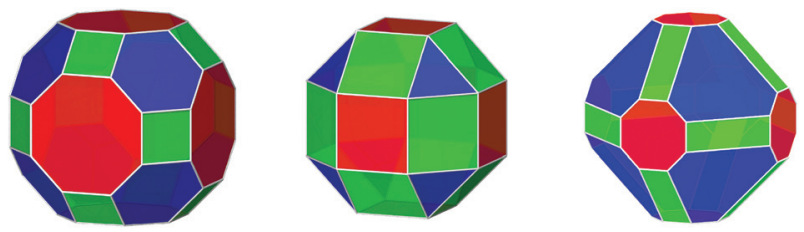

(a)

(b)

(c)

Fig. 1 Compound shapes including (a) the great rhombicuboctahedron (GRO), (b) the small rhombicuboctahedron (SRO), and (c) the modified truncated octahedron (mTO), each enclosed by $\{100\}$ (red), $\{110\}$ (green) and $\{111\}$ (blue) facets.

$\{111\}$ surface area (Fig. 1(c)). In all of the following results and analysis, the $\{100\},\{110\}$ and $\{111\}$ facets, and shapes dominated by them, are encoded with red, green and blue color channels.

Once the nanoparticle shapes and protoparticle meshes are constructed, the nanoparticles in our simulations were dispersed in various ways. For each nanoparticle shape, we have simulated 5800 nanoparticles monodispersed at $2.7 \AA$, with the size distributions indicated in Table 1 . In this way, we can model compound shapes with a mixture of facets, compare the aggregation of different shapes (simulations 1,2 and 3) and investigate the impact of size distributions on each shape ( 1 and 4, 2 and 5, 3 and 6). These results go well beyond the demonstrative Platonic and Catalan solids presented in ref. 41. Future work is planned to investigate the impact of mixing the three shapes in various ways.

\section{Sample computation}

Two force fields are employed within the MD simulation, as described in detail elsewhere. ${ }^{41}$ The first is a harmonic potential between neighbouring protoparticles on the same nanoparticle used to preserve nanoparticle shape. The primary parameter is the spring constant which determines the stiffness of the protoparticle mesh with respect to deformation. A value of $320 \mathrm{~N} \mathrm{~m}^{-1}$ (reduced unit spring constant of 20) was used which is approximately on the order of the spring constant for molecular bonds. The second force field is a Morse potential used to model the interaction between pairs of protoparticles on two different nanoparticles. Since the nanodiamonds in this work have three types of facets, $\{100\}$,

Table 1 Specifics of the shapes and size distributions used in the simulations described herein. All simulations chain a total of 5800 nanoparticles in a simulation supercell of $290000 \mathrm{~nm}^{3}$, giving a density of $2.0 \times 10^{19}$ nanoparticles per $\mathrm{m}^{3}$

\begin{tabular}{lllllll}
\hline Fig. & $2.2 \mathrm{~nm}$ & $2.7 \mathrm{~nm}$ & $3.2 \mathrm{~nm}$ & GRO & SRO & mTO \\
\hline 2(a) & 0 & 5800 & 0 & 5800 & 0 & 0 \\
2(b) & 0 & 5800 & 0 & 0 & 5800 & 0 \\
2 (c) & 0 & 5800 & 0 & 0 & 0 & 5800 \\
$2(\mathrm{~d})$ & 1160 & 2900 & 1740 & 5800 & 0 & 0 \\
$2(\mathrm{e})$ & 1160 & 2900 & 1740 & 0 & 5800 & 0 \\
2(f) & 1160 & 2900 & 1740 & 0 & 0 & 5800
\end{tabular}

$\{111\}$ and $\{110\}$, there are a total of six unique interactions and therefore six sets of parameters are required for this potential.

In the present study, these are obtained via Density Function Tight Binding (DFTB) calculations of the separationand orientation-dependent potential energy binding curve between pairs of atomistic nanodiamond models, ${ }^{49,50}$ but they can similarly be obtained from any reliable electronic structure or classical computational method. The atomistic nanoparticles were unpassivated and as such, contained facet dependent couloumbic interactions which are in contrast to passivated nanodiamonds. ${ }^{50}$ These facet-facet curves were then converted to the point to point interaction parameters for the surface mesh of protoparticles via a fitting Monte Carlo routine prior to the MD simulation, as describe elsewhere. ${ }^{40,41}$ The potential is smoothly truncated between $4 \AA$ and $6 \AA$ using a quadratic damping function.

A Verlet integration algorithm with a time step of 3 fs was employed using a simple velocity rescaling thermostat. The temperature, $T$, in SNAP can be defined using either the nanoparticle centre of mass velocities or the protoparticle velocities and both of these are approximately equal after sufficient equilibration due to energy equipartition. However, due to the coarse-grained nature of the protoparticle mesh, the numerical values of temperature are not physical and we, therefore, use the term pseudo-temperature to refer to this quenching parameter. Additionally, similar care must be taken while interpreting time scales. Although the order of the time-dependent results is still valid, their absolute values may not be entirely accurate. The simulations are performed in a vacuum. This results in very distinct facet dependent interactions which are diminished in solutions as the surfaces become passivated. The number density was selected to conform with experimental observations. ${ }^{51}$

\section{Sample analysis}

The SNAP analyser module provides several tools for characterising the energy, orientation and position of nanoparticles using the MD trajectory and the final frame. Since nanodiamond aggregates are potential drug delivery platforms, and drug loading depends sensitively on the availability of certain facets and the porosity of the system, we have calculated the inter-particle probabilities, the interfacial probabilities with the SNAP analyser along with the nanoparticle coordination. The pore size and pore size distributions were calculated using another compatible package PorosityPlus, which is also available for download. ${ }^{52}$ The Lindemann index was calculated using a development code that will be released in the future.

The inter-particle probability is calculated using the first nearest neighbour coordination distribution, and decomposition of this coordination is based upon nanoparticle sizes and shapes in polydispersed simulations. A cut-off distance which corresponds to the minimum between the first and second peaks in the radial distribution (another property calculated by SNAP) was chosen in each case. The interfacial probability is determined by counting each protoparticle participating in a facet and recording the closest protoparticle on a 
neighbouring nanoparticle within the attractive distance of the Morse potential. Both procedures are described in ref. 40 and 41.

The Lindemann index is a simple measure of the mobility of nanoparticles in the system and is often used to find the transition between the solid and liquid phases. It represents the time-averaged root-mean-square of the relative displacement of nanoparticles. The Lindemann Criterion states that solids have a value of approximately below 0.1 due to the relatively small displacements from vibrations within a solid and liquid are found above this value.

PorosityPlus characterizes the pore geometry within an atomic or particle configuration by calculating the porosity, internal surface area and pore size distribution (PSD) using a variety of Monte Carlo (MC) based integration methods. This begins with random trial point insertions to determine the maximum sphere size which both encompasses the original insertion point at the centre of the sphere but does not overlap any system particles. The software then finds the maximum sphere diameter by a random walkaway from the original insertion point and once found, the point is binned into the sphere diameter histogram at the maximum discovered value. This is repeated over many insertion points to calculate the PSD, as described and demonstrated in ref. 53.

\section{Results and discussion}

The final configurations of the six simulations described above are illustrated in Fig. 2. In all cases, visual inspection reveals the presence of pores, disordered aggregation and ordered domains that have self-assembled. Fig. 2(a), (b) and (c) show the results of the monodisperse GRO, SRO and mTO (respectively) at $2.7 \mathrm{~nm}$. The monodisperse GRO sample (Fig. 2(a)), which appears red due to the dominance of the $\{100\}$ facets in this morphology, exhibits distinctly crystalline domains where the packing of the nanoparticles is almost perfect. These domains are not aligned with respect to each other, but in each case, the inter-particle alignment is the same. These near-perfectly packed regions are not as prevalent when a size distribution is introduced, as we can see in Fig. 2(d). A similar degree of ordering is not evident in the monodisperse SRO (Fig. 2(b)) or the monodisperse mTO (Fig. 2(c)), nor their polydispersed counterparts (Fig. 2(e) and (f), respectively), although some small domains are seen in the monodisperse mTO sample. This clearly indicates that the shape, characterised by the relative area of different crystallographic facets, is important for self-assembly, which appears negatively impacted by size distributions. Each of these issues is analysed in the following sections.

\section{Size distributions}

Considerable effort in the nanoparticle community has been directed to creating samples of nanoparticles that are monodisperse in size. While advances have been made, most samples still retain a narrow size distribution, which has an important impact on the local and global structure of any assemblies. For this reason, it is essential that nanoparticles of various sizes are included in the study of nanoparticle aggregation, and that the relative size differences are comparable with (or ideally identical to) experimental observations. One of the advantages of using SNAP is the ability to represent nanoparticles with realistic sizes, so that the interfacial distances have the correct scale with respect to the nanoparticle diameters. This means that we can simulate different sizes within the one simulation and characterise the distribution of small and large nanoparticles within the final aggregate. In the case of nanodiamonds, this narrow distribution is asymmetrically centred around $2.7 \mathrm{~nm}$ (as mentioned above) with a slightly larger proportion of $>2.7 \mathrm{~nm}$ nanoparticles than $<2.7 \mathrm{~nm}$ nanoparticles, ${ }^{47}$ and this has been reflected in the mixture and distributions summarized in Table 1.

As mentioned above, the inter-particle coordination number is a useful measure of the packing of the nanoparticles and the distribution of different nanoparticles with respect to each other. We performed this analysis on each sample and partitioned the coordination number for each shape into contributions from each size within the mixture. Fig. 3 shows the impact size distributions have on the coordination numbers for (a) GRO nanoparticles, (b) SRO nanoparticles, and (c) mTO nanoparticles, respectively. Here, we can see that most GRO nanoparticles are surrounded by 6 other nanoparticles, which is consistent with the fact that this shape is dominated by $\{100\}$ facets. However, not all the sizes pack the same way. The $2.7 \mathrm{~nm}$ nanoparticles coordinate with 6 other nanoparticles, the larger $3.2 \mathrm{~nm}$ nanoparticles are likely to coordinate with 8 neighbouring nanoparticles, suggesting interfaces involving $\{111\}$ facets, and the smaller $2.2 \mathrm{~nm}$ nanoparticles are likely to coordinate with 5 other nanoparticles. A coordination number of 5 is inconsistent with the symmetry of the GRO suggesting that these nanoparticles may be adjacent to pores, which is consistent with visual inspection.

When the shape of the nanoparticle in the sample changed, we observe a different coordination profile, even though each of these shapes is enclosed by the same facets (in the same configuration, but with different areas). The $\{110\}$ dominated SRO nanoparticles (Fig. 3(b)) are coordinated with other 4 or 5 surrounding nanoparticles, driven mostly by the majority of $2.7 \mathrm{~nm}$ nanoparticles in the sample. Once again the larger $3.2 \mathrm{~nm}$ nanoparticles are likely to coordinate with more neighbouring nanoparticles (in this case 6), and the smaller $2.2 \mathrm{~nm}$ nanoparticles are likely to coordinate with fewer other nanoparticles (in this case 4), supporting the hypothesis that smaller nanoparticles line the pores. The $\{111\}$ dominated mTO nanoparticles (Fig. 3(c)) are coordinated with other 5 or 6 surrounding nanoparticles, with $2.7 \mathrm{~nm}$ nanoparticles centred on the same values, larger $3.2 \mathrm{~nm}$ nanoparticles coordinate with 8 neighbouring nanoparticles and the smaller $2.2 \mathrm{~nm}$ nanoparticles mostly coordinate with 4 or 5 other nanoparticles. The trends with respect to the sizes in the 
(a)

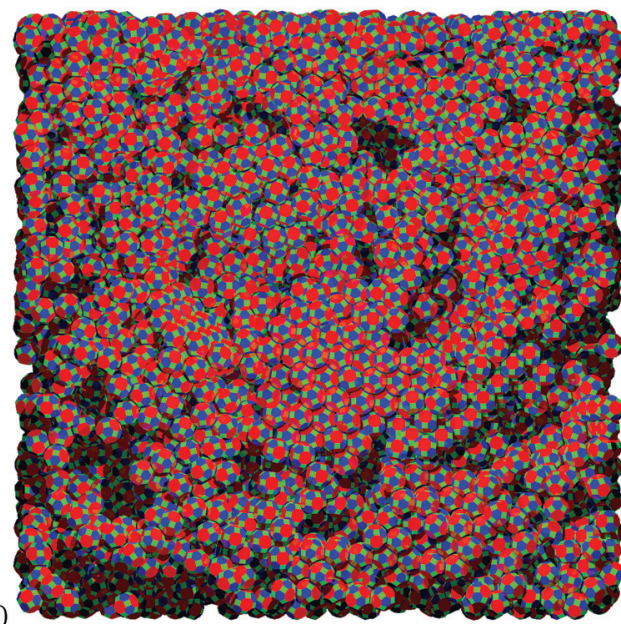

(b)
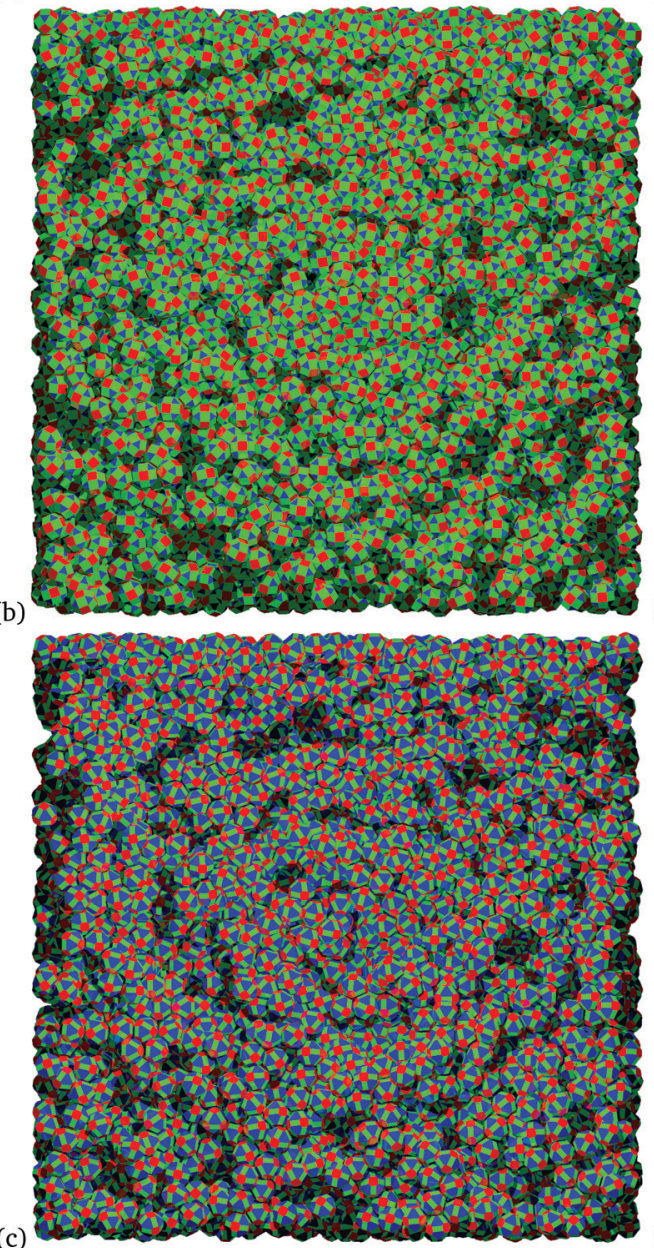

(d)
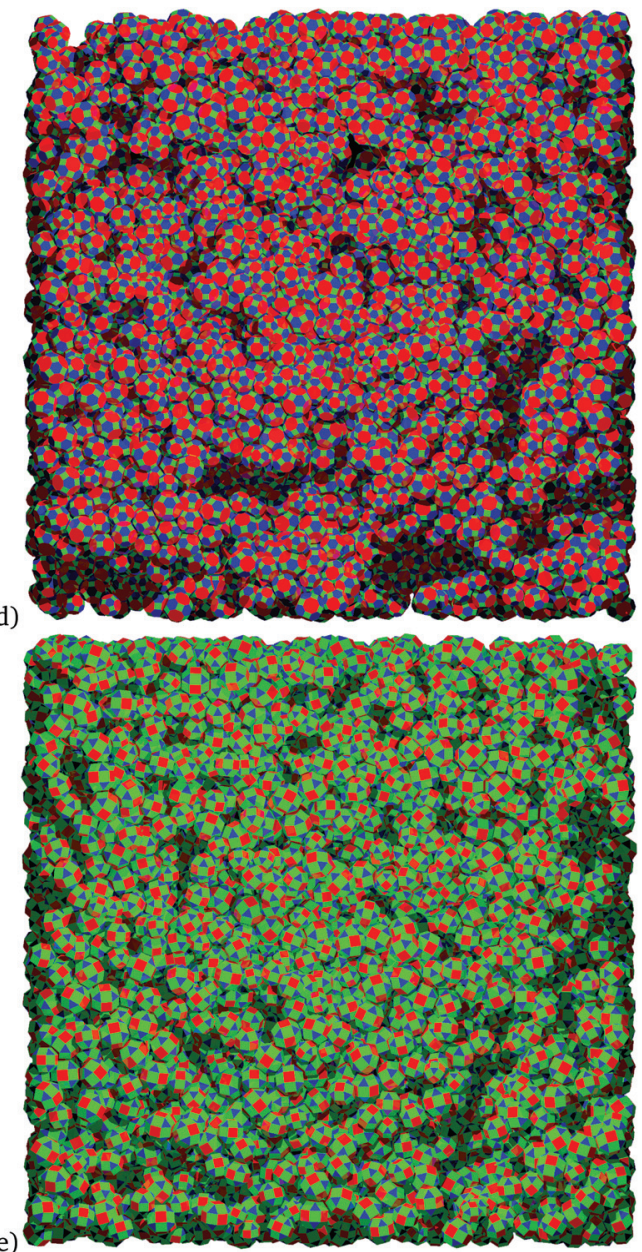

(e)

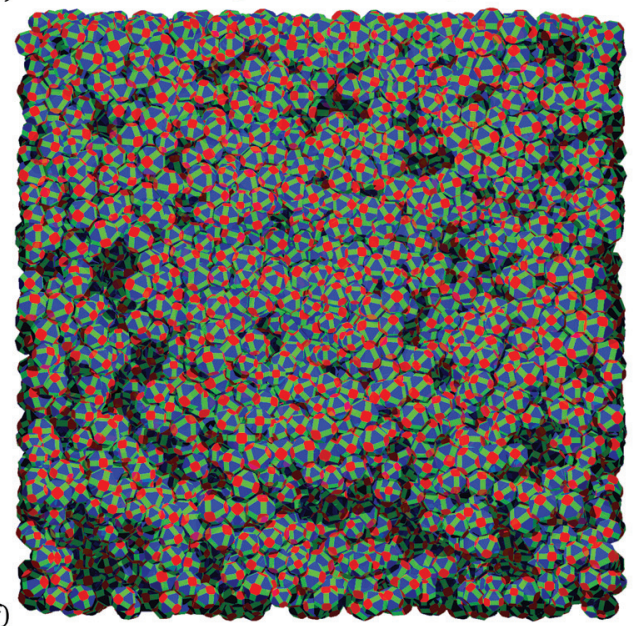

Fig. 2 Examples of the results obtained from the SNAP molecular dynamics simulations for (a) the great rhombicuboctahedron (GRO) at $2.7 \mathrm{~nm}$, (b) the small rhombicuboctahedron (SRO) at $2.7 \mathrm{~nm}$, (c) the modified truncated octahedron (mTO) at $2.7 \mathrm{~nm}$, (d) the GRO with a distribution of sizes, (e) the SRO with a distribution of sizes, and ( $f$ ) the mTO with a distribution of sizes.

samples are entirely consistent, with some clearly shapedependent preferences for different coordination numbers.

To further enlighten the issue of packing among the nanoparticles we have also calculated the relative population of different interfaces present in the aggregates. There are six different types of interfaces possible, given the three shapes, but only three types of interfaces are formed: $\{111\} \mid\{111\}$, $\{100\} \mid\{110\}$ and $\{100\} \mid\{111\}$ (see Fig. 4). The majority of interfaces between GRO nanoparticles involve $\{100\}$ (Fig. 4(a)), which is consistent with this being the dominant facet for this 


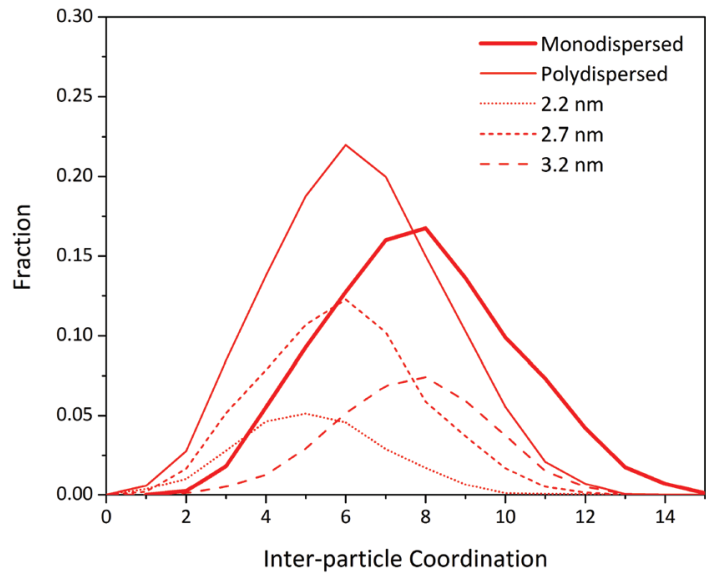

(a)

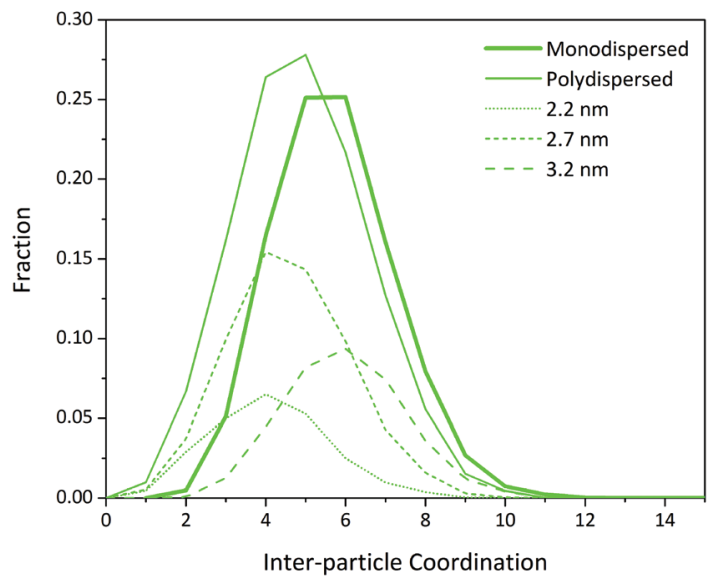

(b)

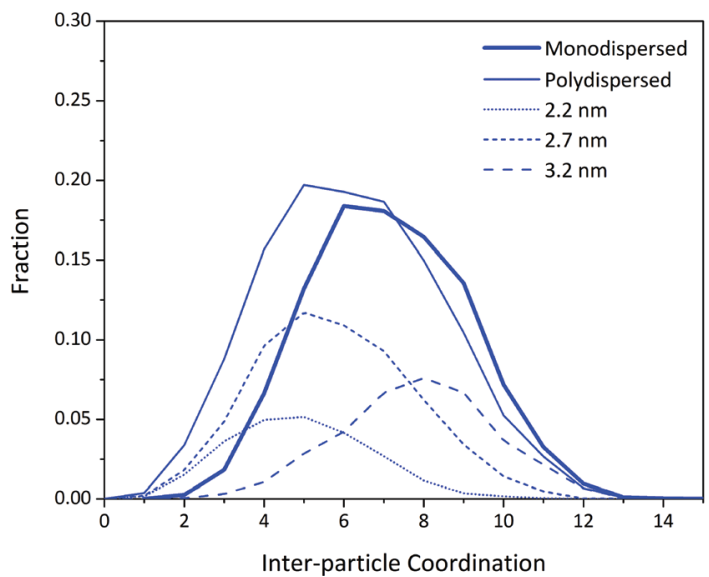

(c)

Fig. 3 Impact of polydispersivity on the coordination numbers for (a) great rhombicuboctahedral nanoparticles (GRO), (b) small rhombicuboctahedral nanoparticles (SRO), and (c) modified truncated octahedral nanoparticles (mTO). The relative magnitude of each type of inter-particle coordination is a reflection of the relative fraction of $2.2 \mathrm{~nm}, 2.7 \mathrm{~nm}$ and $3.2 \mathrm{~nm}$ nanoparticles in the sample (see Table 1).

shape, and the majority of interfaces between mTO nanoparticles involve $\{111\}$ (Fig. $4(\mathrm{c})$ ), which is consistent with this being the dominant facet for this shape. The SRO nano- particles are dominated by $\{110\}$ facets, and while this shape produced more interfaces involving the $\{110\}$ facet than the alternative shapes (Fig. 4(b)), these interfaces do not dominate the sample. Regardless of the shapes and the sizes, the interfaces between nanoparticles are always dominated by $\{111\}$ facets, which corresponds to the deep wells in the Morse potential for these interactions as detailed in the ESI. $\dagger$ This is also supported experimentally as shown in Fig. 4(e). Here, we see the cryo-TEM image of a representative nanodiamond aggregate with dominating interfaces of $\{111\} \mid\{100\}$ and $\{111\} \mid$ $\{111\}$, indicated by the arrows. The red arrows indicate the presence of $\{111\}$ lattice fringes of a diamond. The image was taken using a cryo-TEM, Krios with a direct electron camera, operating at $300 \mathrm{kV}$. The TEM specimen was made by plungefreezing of nanodiamond suspensions in water onto a TEM grid. This is significant, as on nanodiamonds these facets are known to graphitize and present as curved aromatic fullerenelike cages $^{44,46}$ that are less reactive to surrounding molecules. ${ }^{54-56}$

\section{Aggregation and self-assembly}

Although the inter-particle coordination and the population of different types of interfaces are useful ways to characterize the local structure of nanoparticle aggregates, it is also insightful to investigate the dynamical time-line of self-assembly to produce long range order. For this task, we have calculated the Lindemann index over the entire length of the MD simulation for each sample as a function of time, as shown in Fig. 5 for (a) the GRO nanoparticles, (b) the SRO nanoparticles, and (c) the mTO nanoparticles, respectively. Each figure includes the monodisperse and the polydispersed samples; the latter has been partitioned into contributions from each of the sizes in the distribution.

From Fig. 5(a) and (c), we can see that the GRO nanoparticles and the mTO nanoparticles exhibit almost identical ordering behavior, packing into more ordered superstructures at approximately the same stage of the quench. Polydispersed samples are slower to order than the monodisperse counterparts, but the overall relationship is independent of the size in the distribution. This is due to the size-dependent mobility observed in the simulations whereby the largest nanoparticles order first, which leads to less efficient packing and more excluded volume than for the monodisperse systems. The SRO nanoparticles, however, are different, as shown in Fig. 5(b). This shape moves from a liquidlike superstructure to a solid-like superstructure at a later stage in the quench compared to the other shapes, but once initiated, the transformation proceeds at the same rate. Polydispersivity is not as important for this shape; the difference between the monodisperse and polydispersed samples is significantly less than in other shapes, as is the difference between the smaller and larger nanoparticles within the sample.

In general, regardless of the shape, the smaller nanoparticles remain more mobile for longer than the larger nanoparticles. This tends to result in some size segregation with the larger nanoparticles tending to pack together first before the medium and finally the smallest nanoparticles losing their 


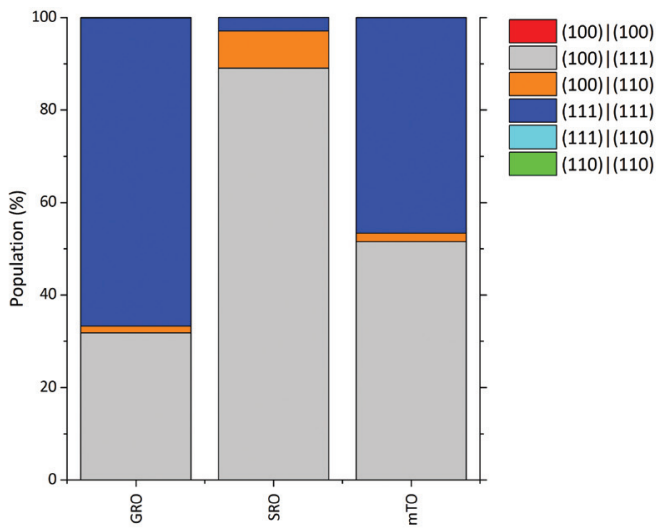

(a)

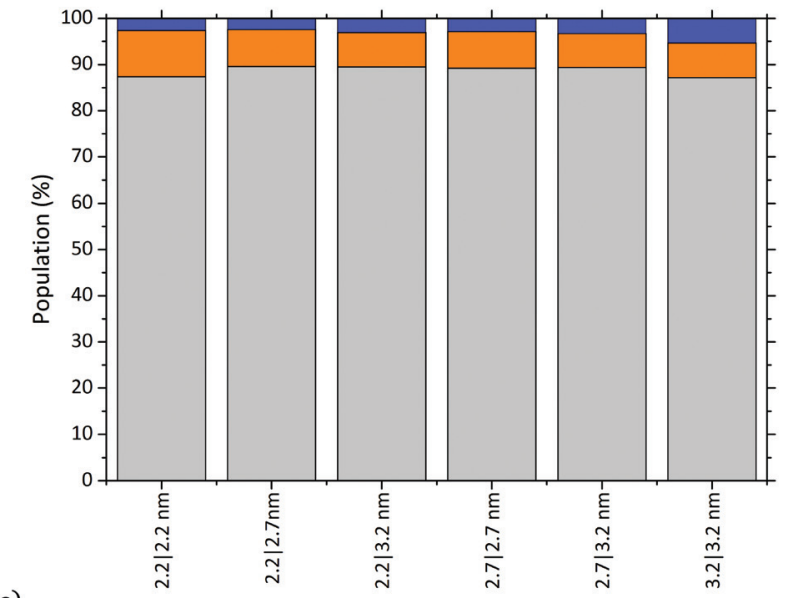

(b)

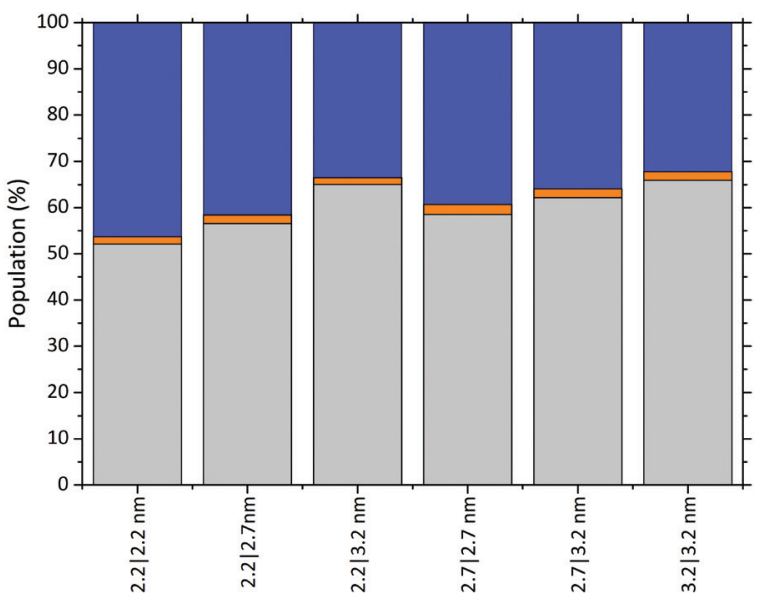

(d)
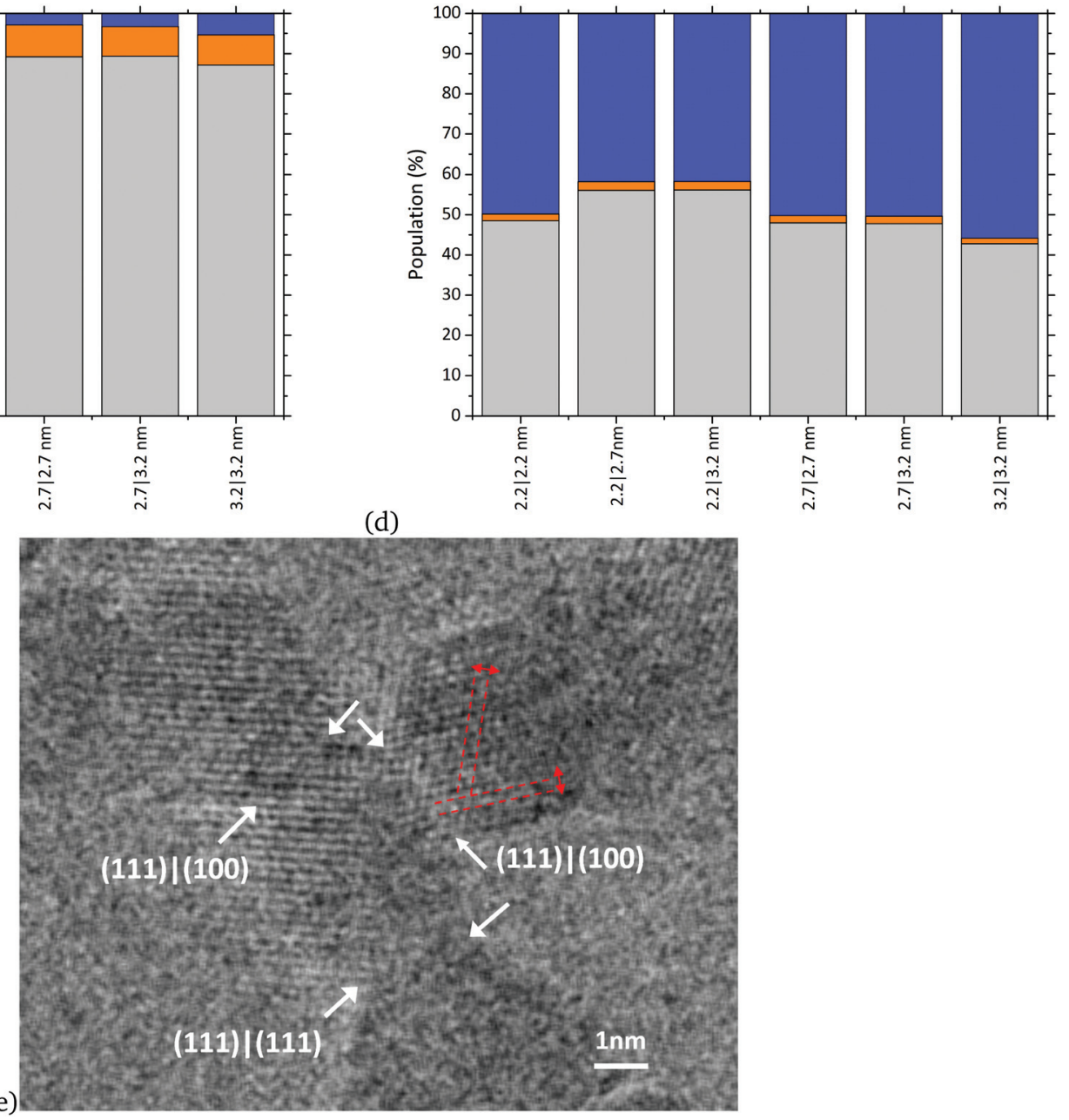

Fig. 4 Impact of size on the interfacial probabilities for the monodisperse (a) great rhombicuboctahedral (GRO), small rhombicuboctahedral (SRO) and modified truncated octahedral (mTO) nanoparticles at $2.7 \mathrm{~nm}$, (b) GRO nanoparticles with different sizes, (c) SRO nanoparticles with different sizes, and (d) mTO nanoparticles with different sizes. The legend in (a) applies to all histograms. Note that $\{100\} \mid\{100\}$ interfaces are typically zero, whereas $\{111\} \mid\{110\}$ and $\{110\} \mid\{110\}$ interfaces are less than $1 \%$. (e) Cyro-TEM image of a representative nanodiamond aggregate showing the dominating interfaces (indicated by arrows). The red arrows indicate the $\{111\}$ lattices of the diamond.

mobility. This size-dependent mobility is caused by the stronger attraction due to the larger facets containing more interacting protoparticles. The smaller nanoparticles remain more mobile because their smaller number of interacting protoparticles lowers the energy barrier for separation, particularly at higher pseudo-temperatures. This observation is supported 


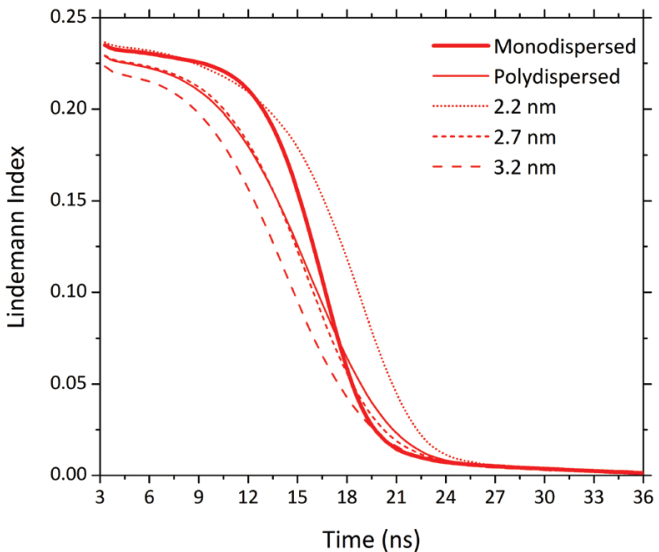

(a)

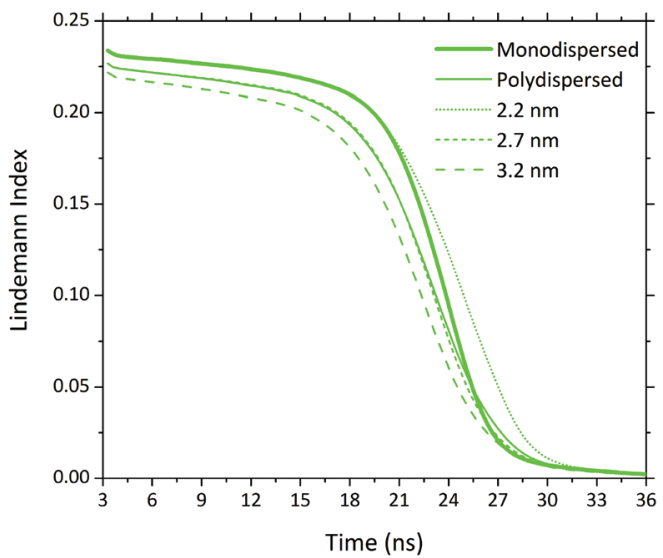

(b)

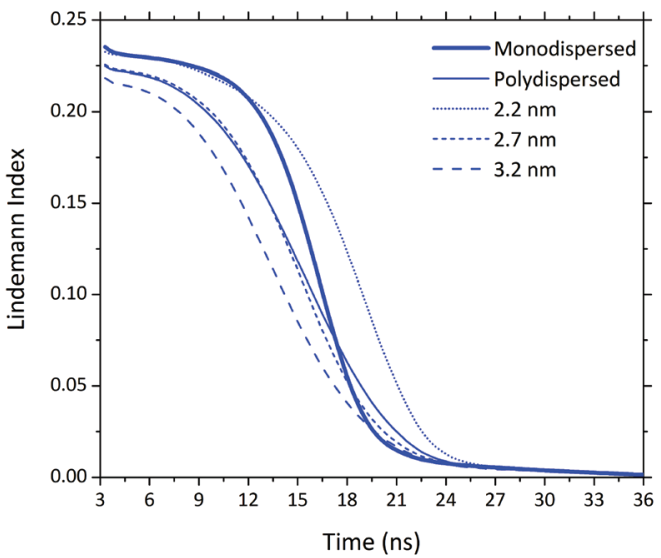

(c)

Fig. 5 Impact of polydispersivity on the Lindemann index for (a) great rhombicuboctahedral (GRO) nanoparticles, (b) small rhombicuboctahedral (SRO) nanoparticles, and (c) modified truncated octahedral (mTO) nanoparticles, as an indicator of the degree of self-assembly. The liquidlike to solid-like transformation boundary is traditionally located at a value of 0.1 (liquid-like above 0.1 , and solid-like below 0.1 ).

experimentally via nanodiamond suspensions in water, ${ }^{57}$ where it was observed that from a distribution of nanoparticle sizes, it was the larger nanoparticles that formed chains while

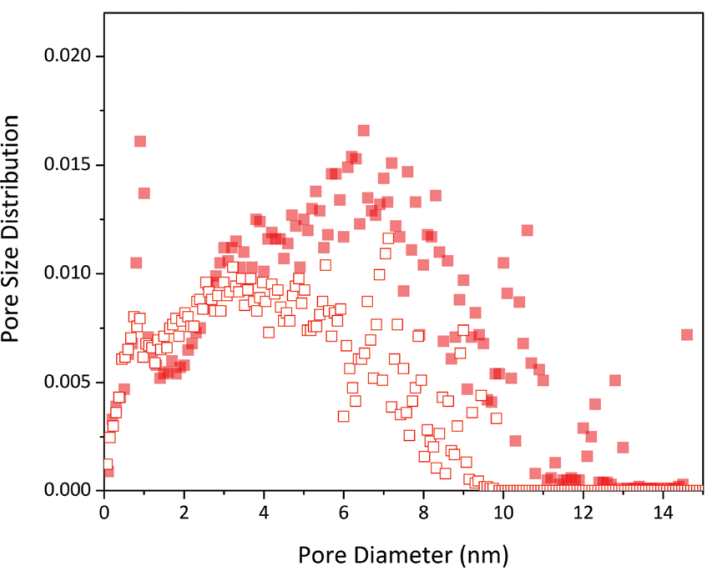

(a)

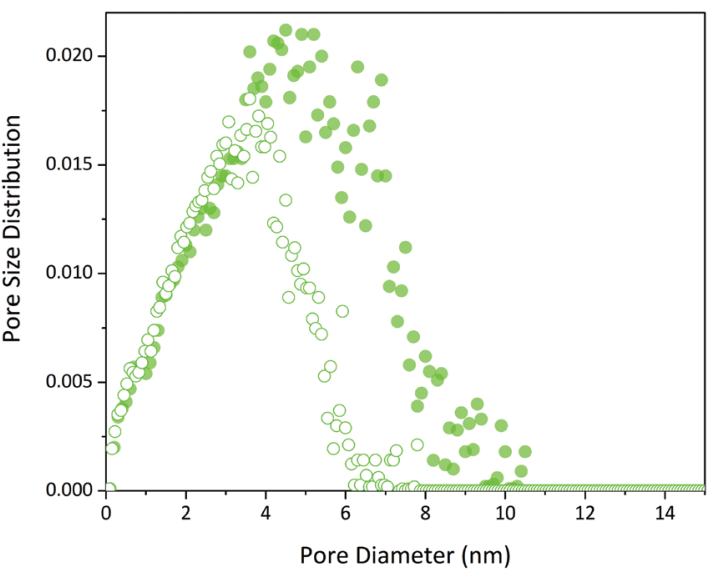

(b)

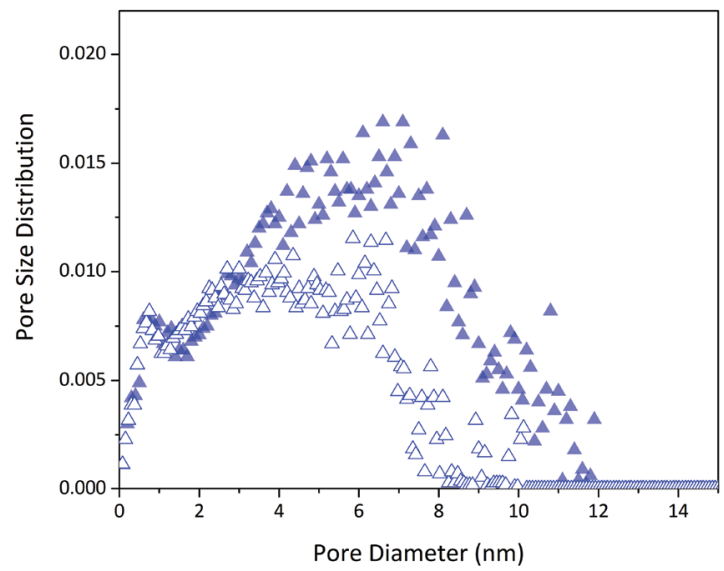

(c)

Fig. 6 Impact of polydispersivity on the pore size and presize distribution of (a) great rhombicuboctahedral (GRO) nanoparticles, (b) small rhombicuboctahedral (SRO) nanoparticles, and (c) modified truncated octahedral (mTO) nanoparticles. Both monodisperse (open symbols) and polydispersed (closed symbols) samples are shown.

the smaller nanoparticles tended to only take part in clusters. Within our simulation, these larger nanoparticles would form an initial stable aggregate backbone for the subsequent packing of the smaller nanoparticle sizes. 


\section{Porosity and pore size distributions}

A common theme that has arisen many times during the analysis described above is the impact of porosity. This is an important issue since nanoporous aggregates are functional primarily because the pores can capture, store or deliver small molecules. In the case of nanodiamonds, the efficacy of drug delivery platforms is strongly influenced by the porosity of the samples, as the size and distribution of the pores is an important design parameter in tuning the doses and dose rates for different therapies. ${ }^{18,20,23-26}$

Fig. 6 provides the results of the pore size and the pore size distribution of (a) the GRO nanoparticles, (b) the SRO nanoparticles, and (c) the mTO nanoparticles, respectively. Each figure includes the monodisperse and the polydispersed samples; the former is shown using open symbols, and the latter by closed symbols. We can see from Fig. 6(b) that the SRO produces smaller pores, with a larger pore size distribution than the other shapes. This is not evident from the visual inspection of Fig. 2(a)-(c) but corroborates the other local and global characterisation methods discussed above. In all cases, the impact of introducing a distribution of sizes is to increase both the size of the pore and the pore size distribution, i.e. to reduce the packing efficiency. This indicates that producing monodisperse samples, either through selective synthesis or via post-processing, will reduce the capacity of nanoporous nanodiamond aggregates to carry drugs and other functional moieties.

Published work on experimental equivalents for the simulated systems is impossible to find but a recent study on nitrogen adsorption isotherms of detonation produced nanodiamonds, which resulted in an average nanoparticle diameter of $4.5+-0.7 \mathrm{~nm}$, has found that the porosity is dominated by nanoparticle sized mesopores of $4-5 \mathrm{~nm}$ in size along with a small population of micropores. ${ }^{58}$ While their experimental samples were at a higher nanoparticle density $\left(2.2 \times 10^{19}\right.$ nanoparticles per $\mathrm{m}^{3}$ compared to $2.0 \times 10^{19}$ nanoparticles per $\mathrm{m}^{3}$ in the simulations), the PSD are similar including the micropore shoulder at small diameters.

\section{Conclusions}

Using the new Simulated Nanoparticle Assembly with Protoparticles (SNAP) package, we have investigated the impact of size distributions on the meso-structure of aggregated faceted polyhedral nanodiamonds in a vacuum. We have reported on three different compound morphologies enclosed by different fractions of low index $\{100\},\{110\}$ and $\{111\}$ facets, known to exhibit positive, near-neutral and negative surface electrostatic potential, respectively. We have compared simulations of monodisperse $(2.7 \mathrm{~nm})$ nanodiamonds with polydispersed samples containing three different sizes in each case. We find that the porosity and packing efficiency can potentially be controlled by tuning the polydispersivity, rather than eliminating it, but the smaller nanoparticles are likely to decorate the inside of pores regardless of the nanoparticle shape.
Additionally, the multi-polar facet-dependent surface electrostatic potential, which is a unique feature of nanodiamonds, has an important effect on the availability of surface facets lining the inside of pores, as well as the overall ordering of the aggregate. Nanoparticles dominated by near-neutral facets form more disordered aggregates, even though closed packed lattices are possible. This indicates that deliberate mixtures of shapes may provide a path to engineering the meso-structure of nanodiamond aggregates, regardless of the size distributions. Additionally, solution-based assembly with surface functionalization is a topic of future work.

\section{Conflicts of interest}

There are no conflicts to declare.

\section{Acknowledgements}

Computational resources for this project have been supplied by the National Computing Infrastructure (NCI) national facility under partner Grant q27.

\section{References}

1 E. M. Hotze, T. Phenrat and G. V. Lowry, J. Environ. Qual., 2010, 39, 1909.

2 M. L. Eggersdorfer and S. E. Pratsinis, Adv. Powder Technol., 2014, 25, 71.

3 F. Gambinossi, S. E. Mylon and J. K. Ferri, Adv. Colloid Interface Sci., 2015, 222, 332.

4 E. Ōsawa, Diamond Relat. Mater., 2007, 16, 2018.

5 O. A. Williams, J. Hees, C. Dieker, W. Jager, L. Kirste and C. E. Nebel, ACS Nano, 2010, 4, 4824.

6 K. Xu and Q. J. Xue, Phys. Solid State, 2004, 46, 649.

7 L. K. Limbach, Y. C. Li, R. N. Grass, T. J. Brunner, M. A. Hintermann, M. Muller, D. Gunther and W. J. Stark, Environ. Sci. Technol., 2005, 39, 9370.

8 J. A. Brant, J. Labille, C. O. Robichaud and M. Wiesner, J. Colloid Interface Sci., 2007, 314, 281.

9 T. Phenrat, N. Saleh, K. Sirk, R. D. Tilton and G. V. Lowry, Environ. Sci. Technol., 2007, 41, 284.

10 L. K. Limbach, R. Bereiter, E. Muller, R. Krebs, R. Glli and W. J. Stark, Environ. Sci. Technol., 2008, 42, 5828.

11 J. Jiang, G. Oberdorster and P. Biswas, J. Nanopart. Res., 2009, 11, 77.

12 D. Jassby, J. F. Budarz and M. Wiesner, Environ. Sci. Technol., 2012, 46, 6934.

13 Y. Shen, C. Wu, T. Q. P. Uyeda, G. R. Plaza, B. Liu, Y. Han, M. S. Lesniak and Y. Cheng, Theranostics, 2017, 7, 1735.

14 A. Molza, J.-L. Mansot, M. J.-F. Guinel and L. Legras, Microsc. Microanal., 2014, 20, 1500.

15 A. Albanese and W. C. W. Chan, ACS Nano, 2011, 5, 5478.

16 Y. H. Hu, O. A. Shenderova, Z. Hu, C. W. Padgett and D. W. Brenner, Rep. Prog. Phys., 2006, 69, 1847. 
17 H. Yockell-Lelievre, F. Lussier and J.-F. Masson, J. Phys. Chem. C, 2015, 119, 28577.

18 H. Huang, E. Pierstorff, E. Ōsawa and D. Ho, Nano Lett., 2007, 7, 3305.

19 H. Huang, E. Pierstorff and D. Ho, ACS Nano, 2008, 2, 203.

20 R. Lam, M. Chen, E. Pierstorff, H. Huang, E. Ōsawa and D. Ho, ACS Nano, 2008, 2, 2095.

21 E. Chow, E. Pierstorff, G. Cheng and D. Ho, ACS Nano, 2008, 2, 33.

22 A. Smith, E. Robinson, X. Zhang, E. Chow, E. Ōsawa and D. Ho, Nanoscale, 2011, 3, 2844.

23 V. M. Mochalin, A. Pentecost, X.-M. Li, I. Neitzel, M. Nelson, C. Wei, T. He, F. Guo and Y. Gogotsi, Mol. Pharmaceutics, 2013, 10, 3728.

24 R. Shimkunas, E. Robinson, X. Zhang, R. Lam, X. Xu, E. Ōsawa and D. Ho, Biomaterials, 2009, 30, 5720.

25 X.-Q. Zhang, M. Chen, R. Lam, X. Xu, E. Ōsawa and D. Ho, ACS Nano, 2009, 3, 2609.

26 H.-J. Kim, K. Zhang, L. Moore and D. Ho, ACS Nano, 2014, 8, 2998.

27 D. Grasso, K. Subramaniam, M. Butkus, K. Strevett and J. Bergendahl, Rev. Environ. Sci. Bio/Technol., 2002, 1, 17.

28 D. Segets, R. Marczak, S. Schäfer, C. Paula, J. F. Gnichwitz, A. Hirsch and W. Peukert, ACS Nano, 2011, 5, 4658.

29 H. H. Liu, S. Surawanvijit, R. Rallo, G. Orkoulas and Y. Cohen, Environ. Sci. Technol., 2011, 45, 9284.

30 K. A. D. Guzman, M. P. Finnegan and J. F. Banfield, Environ. Sci. Technol., 2006, 40, 7688.

31 M. Alimohammadi and K. A. Fichthorn, Nano Lett., 2009, 9, 4198.

32 S. Markutsya, S. Subramaniam, R. D. Vigil and R. O. Fox, Ind. Eng. Chem. Res., 2008, 47, 3338.

33 M. Smith and T. Matsoukas, Chem. Eng. Sci., 1998, 53, 1777.

34 K. Lee and T. Matsoukas, Powder Technol., 2000, 110, 82.

35 H. Zhao, A. Maisels, T. Matsoukas and C. Zheng, Powder Technol., 2007, 173, 38.

36 T. Kim, C. H. Lee, S. W. Joo and K. Lee, J. Colloid Interface Sci., 2008, 318, 238.

37 Z. M. Sherman and J. W. Swan, ACS Nano, 2016, 10, 5260.
38 Z. Zhang and S. C. Glotzer, Nano Lett., 2004, 4, 1407.

39 C. L. Phillips, J. A. Anderson and S. C. Glotzer, Phys. Procedia, 2014, 53, 75.

40 G. Opletal, M. Golebiewski and A. S. Barnard, Simulated Nanoparticle Assembly with Protoparticles (SNAP), v1. CSIRO Software Collection, 2020, DOI: 10.25919/5e5c662a0597e.

41 G. Opletal, M. Golebiewski and A. S. Barnard, JPhys Mater., 2020, 3, 026001.

42 A. Kruger, F. Kataoka, M. Ozawa, T. Fujino, Y. Suzuki, A. E. Aleksenskii, A. Y. Vul' and E. Ōsawa, Carbon, 2005, 43, 1722.

43 H. Huang, L. Dai, D. H. Wang, L.-S. Tan and E. Ōsawa, J. Mater. Chem., 2008, 18, 1347.

44 A. S. Barnard and M. Sternberg, J. Mater. Chem., 2007, 17, 4811.

45 E. Ōsawa, D. Ho, H. Huang, M. V. Korobov and N. N. Rozhkova, Diamond Relat. Mater., 2009, 18, 904.

46 L.-Y. Chang, E. Ōsawa and A. S. Barnard, Nanoscale, 2011, 3, 958 .

47 J. Hees, A. Kriele and O. A. Williams, Chem. Phys. Lett., 2011, 509, 12.

48 A. S. Barnard and E. Ōsawa, Nanoscale, 2014, 6, 1188.

49 A. S. Barnard, J. Mater. Chem., 2008, 18, 4038.

50 L. Lai and A. S. Barnard, J. Phys. Chem. Lett., 2012, 3, 896.

51 E. Ōsawa, Personal Communication, 31/10/2017.

52 G. Opletal and A. S. Barnard, PorosityPlus. v1. CSIRO. Software Collection, 2018, DOI: 10.25919/5b8e0ffa8afaa.

53 G. Opletal, T. C. Petersen, S. P. Russo and A. S. Barnard, JPhys Mater., 2018, 1, 016002.

54 L. Lai and A. S. Barnard, Nanoscale, 2014, 6, 14185.

55 L. Lai and A. S. Barnard, Nanoscale, 2016, 8, 7899.

56 A. S. Barnard, Nanoscale, 2017, 9, 70.

57 S. L. Y. Chang, P. Reineck, D. Williams, G. Bryant, G. Opletal, S. A. El-Demrdash, P.-L. Chiu, E. Ōsawa, A. S. Barnard and C. Dwyer, Nanoscale, 2020, 12, 5363.

58 E. Pina-Salazar, K. Urita, T. Hayashi, R. Futamura, F. Vallejos-Burgos, J. Wloch, P. Kowalczyk, M. Wisniewski, T. Sakai, I. Moriguchi, A. P. Terzyk, E. Ōsawa and K. Kaneko, Langmuir, 2017, 33, 11180. 